Check for updates

Cite this: RSC Adv., 2017, 7, 34215

Received 31st May 2017

Accepted 30th June 2017

DOI: 10.1039/c7ra06081e

rsc.li/rsc-advances

\section{The effects of central metals on ammonia sensing of metallophthalocyanines covalently bonded to graphene oxide hybrids $\uparrow$}

\author{
Yong Li, ${ }^{a}$ Bin Wang, DD *a Zheying Yu, ${ }^{\text {a }}$ Xiaoqing Zhou, ${ }^{a}$ Di Kang, ${ }^{a}$ Yiqun Wu, ${ }^{\text {ab }}$ \\ Zhimin Chen, ${ }^{a}$ Chunying He ${ }^{a}$ and Xin Zhou ${ }^{* c}$
}

\begin{abstract}
Cost-efficient, highly sensitive, selective and stable sensing materials play a key role in developing $\mathrm{NH}_{3}$ sensors. Herein, a series of tetra- $\beta$-aminephthalocyanines metal(I) (aPcMs $\mathrm{M}=\mathrm{Cu}, \mathrm{Ni}, \mathrm{Co}, \mathrm{Fe}$ ) have been successfully covalently bonded on the surface of graphene oxide (GO) by a facile amidation reaction. The obtained aPcM-GO hybrids display good dispersibility, which is beneficial to construct uniform sensing devices. The aPcM-GO sensors exhibit excellent sensing performance in terms of sensitivity, reversibility, reproducibility, selectivity and stability, especially the aPcCo-GO sensor which exhibited a response of about $11.6 \%(50 \mathrm{ppm})$, a limit of detection as low as $800 \mathrm{ppb}$, and a recovery time of about as fast as $350 \mathrm{~s}$ at room temperature. The enhanced $\mathrm{NH}_{3}$-sensing performance is mainly due to the synergistic effect between aPcM and GO, e.g. the stronger adsorption interaction of aPcM with $\mathrm{NH}_{3}$, the high electrical conductivity of GO, and the fast charge transfer between $\mathrm{APCM}$ and GO. By comparison, the response of aPcM-GO hybrids to ammonia decreases gradually in the following order of $\mathrm{Co}>\mathrm{Cu}>\mathrm{Ni}>\mathrm{Fe} \gg \mathrm{GO}$, indicating that the central metals play a critical role in gas sensitivity toward $\mathrm{NH}_{3}$, which is further confirmed by first-principle density functional theory.
\end{abstract}

\section{Introduction}

With the increase of air pollution and concern about the impact of air quality on human health, global environmental problems caused by the release of chemical pollutants have attracted great attention. ${ }^{1-3}$ Ammonia $\left(\mathrm{NH}_{3}\right)$, a colorless, odorous, toxic and flammable gas, is widely used in agriculture, food industry and industrial chemicals, and comes from industry, automobile emissions and biological metabolism, etc. ${ }^{4}$ When $\mathrm{NH}_{3}$ concentration exceeds $300 \mathrm{ppm}$, it will damage human cells and cause serious injuries in skin, eyes and respiratory tract. ${ }^{1}$ According to the United States Occupational Safety and Health Administration (NIOSH), there is a $15 \mathrm{~min}$ and $10 \mathrm{~h} \mathrm{NH}_{3}$ exposure limit of $35 \mathrm{ppm}$ and 25 ppm in the workplace, respectively. ${ }^{5}$ Therefore, it is significant to develop highly efficient, low power and cost $\mathrm{NH}_{3}$ sensors operated at room temperature for environment protection, industrial production and safety and health monitoring. Cost-

${ }^{a}$ Key Laboratory of Functional Inorganic Material Chemistry, Ministry of Education, School of Chemistry and Materials Science, Heilongjiang University, Harbin 150080, P. R. China.E-mail: wangbin@hlju.edu.cn

${ }^{b}$ Shanghai Institute of Optics and Fine Mechanics, Chinese Academy of Sciences, Shanghai 201800, P. R. China. E-mail: yqwu@siom.ac.cn

${ }^{c}$ MIIT Key Laboratory of Critical Materials Technology for New Energy Conversion and Storage, School of Chemistry and Chemical Engineering, Harbin Institute of Technology, Harbin, P. R. China

† Electronic supplementary information (ESI) available. See DOI: 10.1039/c7ra06081e efficient, highly sensitive and stable sensing materials, for example, semiconductors, ${ }^{6-8}$ conjugated polymers, ${ }^{9-11}$ carbon nanomaterials ${ }^{\mathbf{1 2 - 1 5}}$ and their composite materials, ${ }^{\mathbf{1 6 - 2 0}}$ have been successfully explored to fabricating $\mathrm{NH}_{3}$ sensors. Among them, pristine and modified graphene materials (pristine graphene PG, graphene oxide GO, reduced graphene oxide RGO) are widely used as the most popular sensing materials for gas detection due to its large specific surface area, unique structure and electrical properties such as high electron mobility and low electrical noise. ${ }^{21-26}$ GO is a promising candidate for gas sensing owing to its unique two-dimensional structure, oxygen rich functional groups, common method for graphene synthesis and suitable for chemical modification. ${ }^{23,25}$ Peng et al. also predicted that GO has better $\mathrm{NH}_{3}$ sensing performance than PG because the surface active defect sites of GO (such as the epoxy, hydroxyl and carbonyl groups) can enhance the interactions between ammonia molecules and GO using the first-principles calculations. ${ }^{27}$ However, there are still some drawbacks, such as poor gas-sensing selectivity, low response, slow recovery performance, which limit its practical application. GO with functional molecules is an effective approach to improve the gas sensing performance. The hybridization of metal, metaloxide nanoparticles and polymer with GO have demonstrated a significant synergistic effect towards $\mathrm{NH}_{3}, \mathrm{CO}$, $\mathrm{NO}_{x}$, etc. gas and vapor sensing. ${ }^{28-30}$ Metalphthalocyanines (PcMs) are well-known gas sensing material due to their unique $18 \pi$ conjugated skeleton with diversity of central metal atoms and easily tailored functional groups at the outer of phthalocyanine 


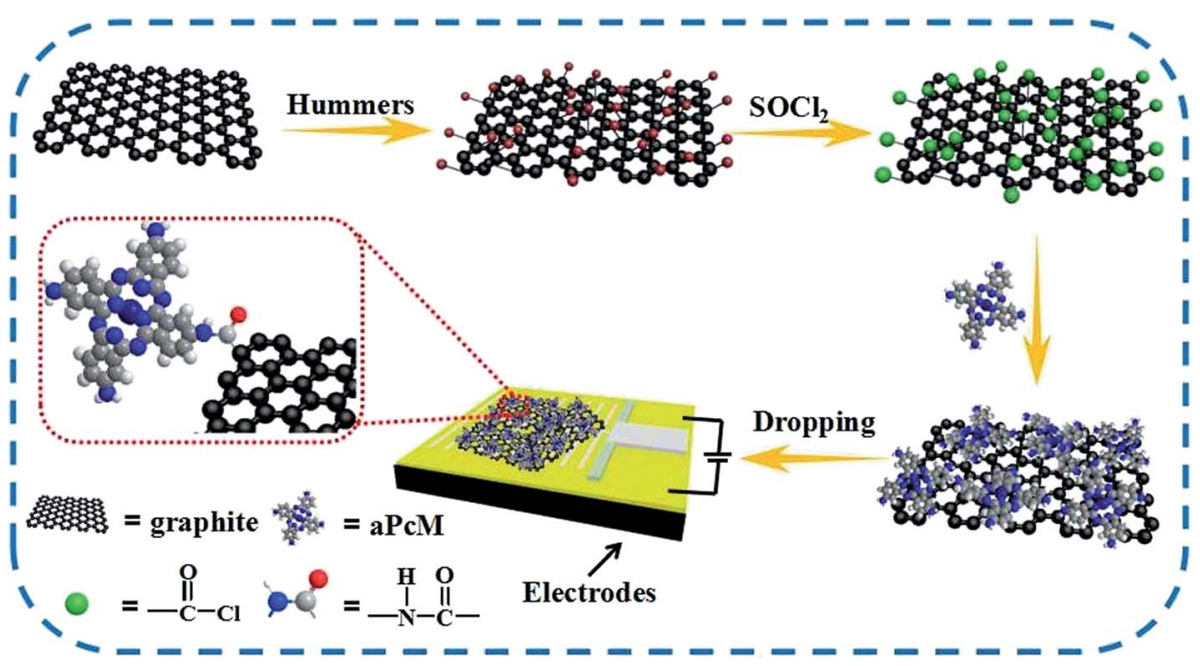

Scheme 1 Schematic of $\mathrm{NH}_{3}$-sensing sensor composed of aPcM-GO hybrids.

ring. ${ }^{31-36}$ Many studies indicated that PcMs based sensors towards $\mathrm{NH}_{3}$ have high sensitivity, quick response and recovery time and good selectivity at room temperature. For example, Ding et al. ${ }^{31}$ revealed that amphiphilic phthalocyanines with central $\mathrm{Zn}$ atom (APcMZn) shows better $\mathrm{NH}_{3}$ sensing performance than amphiphilic phthalocyanines (APcM). Jiang et al. ${ }^{32}$ reported that copper tetra-4-(2,4-di-tert-amylphenoxy) phthalocyanine (tapPcCu) can be used as sensing material for detection of $\mathrm{NH}_{3}$. Recently, we compared the $\mathrm{NH}_{3}$ sensing behavior of tetra-isopentyloxyphthalocyanine with different central atoms and exhibited good response, reversibility, stability and faster response and recovery characteristic to $\mathrm{NH}_{3} \cdot{ }^{37}$ However, low electrical conductivity, small accessible surface area and less exposed active sites for sensing seriously limit the practical application of PcMs in gas sensor. $^{38}$ One effective solution dealing with this issue is to combine the conductive GO to improve the charge transfer of PcM sensors. Owing to the unique two-dimensional structure, large surface area and high conductivity and stability of GO, electrons of PcMs can be easily accepted by GO then be transported quickly. Therefore, the combination of high conductivity and stability of GO with the high reactivity of PcMs sensor is a promising approach to gain better gas sensing performance for gas sensor application. On the one hand, the resistance change due to the charge transfer between PcM/GO hybrids and adsorbed gas molecules becomes easier. On the other hand, PcMs load on the surface of large twodimensional surface area GO, which promotes more PcMs molecules as active sites interacting with gas molecules for high sensitivity. ${ }^{39}$ In fact, PcM/CNT and PcM/RGO hybrids have been self-assembled through $\pi-\pi$ stacking interaction to detect $\mathrm{NH}_{3}$ gas due to the flat, planar aromatic structure of PcMs in previous research. The synergistic effect of PcMs and carbon materials leads to excellent gas sensing performance of PcM/CNT and PcM/RGO hybrids that can be superior to pristine PcM and carbon materials in terms of sensitivity, selectivity, and recovery time. ${ }^{40-42}$ However, due to the indefinable $\pi-\pi$ interactions and van der Waals force between PcMs and carbon materials, it is difficult to control the architecture and properties of hybrids, further resulting in poor gas-sensing reproducibility and stability. Thereby, it is still one of promising research topic to develop PcMs/carbon materials with special structure for high efficient gas sensor. Compared with noncovalent modification, covalent bonding directly to the surface of carbon materials with PcMs can achieve effectively adjusting of the architecture and properties through a chemical coordination method. The covalent modification of PcMs to the carbon materials has been reported for facilitating the combination of PcMs and carbon materials and improving their properties and application. ${ }^{4-45}$ However, GO covalently bonded with PcMs is not explored for gas sensing. Moreover, no systematic study has worked on the effect of central atoms on the gas sensing performance of PcM-GO hybrids.

Based on the above-mentioned drawbacks, in this work we report the facile preparation of a series of tetra- $\beta$-aminephthalocyanines metal covalently bonded to $\mathrm{GO}$ (aPcCu-GO, aPcNi$\mathrm{GO}$, aPcPb-GO and aPcFe-GO) hybrids via an amidation reaction (as show in Scheme 1). A systematic study was done to evaluate the $\mathrm{NH}_{3}$-sensing performance of the hybrids operating in chemiresistive mode over a wide range of concentrations at room temperature (as show in Scheme 1). As expected, the as-prepared aPcM-GO hybrids show an enhanced sensitivity, selectivity, reproducibility, response and recovery time in comparison to other $\mathrm{NH}_{3}$-sensing materials. The results demonstrate that the aPcM-GO hybrids can serve as an excellent $\mathrm{NH}_{3}$ sensing material and have great potential applications for gas sensor. Significantly, the central metals are directly correlated with the $\mathrm{NH}_{3}$-sensing performance. Density functional theory (DFT) calculations were further carried out to reveal the interactions and charge transfer between $\mathrm{NH}_{3}$ and aPcMs with different center metals.

\section{Experimental and calculation details}

\subsection{Reagents}

Flake graphite was purchased from Nanjing XFNANO Materials Tech Co., Ltd. 4-Nitrophthalonitrile (99\% purity) and DBU (98\% 
purity) were purchased from Sigma-Aldrich Co. LLC. Graphene Oxide (GO) was fabricated by the modified Hummers method, which was represented in our previous reports. ${ }^{42}$ Tetra- $\beta$-aminephthalocyanines metal(II) (aPcMs $\mathrm{M}=\mathrm{Cu}(\mathrm{II}), \mathrm{Ni}(\mathrm{II}), \mathrm{Co}(\mathrm{II})$, $\mathrm{Fe}(\mathrm{II}))$ was synthesized by the ordinary template reaction of 4nitrophthalonitrile with anhydrous metal(II) chloride in the presence of 1,8-diazabicyclo-[5,4,0]undec-7-ene (DBU). And then the above products with sodium sulfide reduction (see the ESI for experimental details $\uparrow$ ). Ultrapure water was obtained by using a Millipore Milli-Q system (Millipore Corp. Bedford, MA, USA). All other reagents in this work were of analytical grade and used without further purification.

\subsection{Preparation of aPcM-GO hybrids}

Four aPcM-GO hybrids, via aPcCu-GO, aPcNi-GO, aPcCo-GO and aPcFe-GO, were prepared by the same general method: GO $(20 \mathrm{mg})$ in $\mathrm{N}, \mathrm{N}$-dimethylformamide (DMF) $(20 \mathrm{ml})$ was sonicated at room temperature for $2 \mathrm{~h}$, and then excess thionyl chloride was added. The ensuing mixture was refluxed for $24 \mathrm{~h}$ with continuous stirring under a nitrogen atmosphere. Subsequently, the excess thionyl chloride was removed by distillation. After that, aPcM $(60 \mathrm{mg})$ in DMF $(20 \mathrm{ml})$ with several drops of triethylamine were added and stirred mixture at $100{ }^{\circ} \mathrm{C}$ for $48 \mathrm{~h}$ under vigorous stirring. After cooling, a large of ultrapure water was added. The resultant black solution was filtered through a $0.22 \mu \mathrm{m}$ PTFE membrane filter. The filter cakes were washed thoroughly with tetrahydrofuran (THF) and ethanol, DMF, and acetone, until the filtrate became colorless, and then dried in a vacuum oven for $2 \mathrm{~h}$ at $80{ }^{\circ} \mathrm{C}$ obtaining the desired black aPcM-GO hybrids powders.

\subsection{Sensors assembling and sensing measurement}

A complete description of the gold interdigitated electrodes (IDEs) and the gas sensor testing device was already reported in our previous study. ${ }^{41,42}$

To prepare gas sensors composed of aPcM-GO hybrids, the as-prepared aPcM-GO hybrids were dispersed in DMF to obtain a uniform suspension of $0.5 \mathrm{mg} \mathrm{ml}^{-1}$ by ultrasonication for $2 \mathrm{~h}$, and then the homogeneous dispersion was drop cast onto the gold electrodes using a microsyringe until a resistance range (about 0.1-0.2 M $\Omega$ ) was achieved. Low concentration dispersion was used to avoid overlapping aPcM-GO hybrids. To remove the solvent of the sensor and improve the electrical contact between the hybrids and the gold electrodes, the sensor devices were annealed in a vacuum oven for $2 \mathrm{~h}$ at $80^{\circ} \mathrm{C}$ before sensing tests. For comparison, gas sensor of free GO and aPcM was also fabricated by the similar procedures.

A typical sensing test cycle consisted of three sequential steps. The sensors were dried in a vacuum oven for $1 \mathrm{~h}$ at $80{ }^{\circ} \mathrm{C}$ before measuring. First, a dry air flow was introduced into the sensing test chamber to record a baseline. Then, an appropriate amount of the dry $\mathrm{NH}_{3}$ sample gas was injected to register sensor signals. Finally, the sensor was recovered in a dry air flow. In order to study the effect of relative humidity on the $\mathrm{NH}_{3}$ response properties, dry air and air with controlling different humidity were used as both a carrier and a dilution gas, respectively, and all measurements were performed at $28 \pm$ $0.5^{\circ} \mathrm{C}$. The change in resistance of the sensors was measured with a CUST G2 gas sensing test system (advanced sensor technology laboratory of Jilin university, China). In this study, response is defined by the relative resistance change, as follows:

$$
\text { Response }(\%)=\frac{\Delta R}{R_{\mathrm{a}}} \times 100 \%=\frac{R_{\mathrm{g}}-R_{\mathrm{a}}}{R_{\mathrm{a}}} \times 100 \%
$$

where $R_{\mathrm{a}}$ and $R_{\mathrm{g}}$ were the sensor resistance in initial air flow which was used as background and the target gas. Response and recovery times were defined as the time needed for $90 \%$ of total resistance change on exposure to target gas and air, respectively. High pure $\mathrm{NH}_{3}$ gas was used as the $\mathrm{NH}_{3}$ source. High pure $\mathrm{NH}_{3}$ gas (99.9\%) was purchased from Guangming Research and Design Institute of Chemical Industry, PR China. $\mathrm{NH}_{3}$ gas is stored in a dedicated high-pressure gas cylinder. $\mathrm{NH}_{3}$ sample gas was purged into an anticorrosive air bag consisted of PET/Al/CPP composite membrane through a dry tube filled with $\mathrm{NaOH}$ flakes, to obtain dry $\mathrm{NH}_{3}$ sample gas.

\subsection{Characterization}

UV-vis absorption spectra were recorded with a Lambda 35 UVvis spectrometer (Perkin-Elmer, USA). The Raman spectra were acquired by using a Raman spectrophotometer (HR800, HORIBA Jobin Yvon Company) excited by a laser with $457.9 \mathrm{~nm}$ wavelength. XPS were acquired on a Kratos AXIS Ultra DLD system by using monochromated $\mathrm{Al} \mathrm{K} \alpha \mathrm{X}$-ray beams as the excitation source $(1486.6 \mathrm{eV})$. The base pressure of the analysis chamber was below $10^{-9}$ mbar. Binding energies were calibrated relative to the $\mathrm{C} 1 \mathrm{~s}$ peak at $284.6 \mathrm{eV}$. Raman spectroscopy was performed on a Jobin Yvon HR800 Raman spectrometer by using a $458 \mathrm{~nm}$ laser source. Scanning electron microscopy (SEM) images were recorded with a Hitachi S-4800 field emission scanning electron microscope operating at $15 \mathrm{kV}$. Samples were drop-deposited onto the interdigitated electrodes and measured directly. Transmission electron microscopy was performed with a JEM 2100 instrument at $200 \mathrm{kV}$ utilizing a JEOL FasTEM system. Samples were dropped onto $\mathrm{Cu}$ grids with lacey carbon film and allowed to dry thoroughly before imaging. The current-voltage $(I-V)$ characteristics of the fabricated sensors were measured using a Keithley 4200 semiconductor parameter analyzer with a two-point probe setup via sweeping the potential between -2 and $+2 \mathrm{~V}$ under a $0.01 \mathrm{~V} \mathrm{~s}^{-1}$ scan rate.

\subsection{Calculation details}

DFT calculations were performed for the adsorption of $\mathrm{NH}_{3}$ on PcMs using long-range corrected functional of CAM-B3LYP ${ }^{46}$ with a set of hybrid basis sets (LanL2DZ for metals and 6-31g(d) for $\mathrm{H}, \mathrm{C}$, and N). Charge analyses were performed with the NBO (natural bond orbital) method. ${ }^{47}$ Adsorption energy $\left(E_{\text {ads }}\right)$ between $\mathrm{NH}_{3}$ and the PcMs was defined as,

$$
E_{\text {ads }}=E\left(\mathrm{NH}_{3}+\mathrm{PcMs}\right)-E(\mathrm{PcMs})-E\left(\mathrm{NH}_{3}\right)
$$

where $E($ molecule $+\mathrm{PcMs})$ is the total energy of the $\mathrm{NH}_{3}-\mathrm{PcMs}$, and $E(\mathrm{PcMs})$ and $E\left(\mathrm{NH}_{3}\right)$ are the total energy of $\mathrm{PcMs}$ and $\mathrm{NH}_{3}$, 
respectively. The Gaussian 09 quantum chemical package was employed for all calculations in the present work. ${ }^{48}$

\section{Results and discussion}

\subsection{Characterization of aPcM-GO hybrids}

According to our strategy, aPcM can be covalently bonded to GO by a facile reaction and aPcM-GO hybrids are obtained, which are confirmed by IR spectra, UV-vis spectra, Raman spectroscopy, XPS, SEM and TEM. The FT-IR spectra of GO, aPcCu and aPcCuGO hybrid are shown in Fig. 1A. aPcCu shows characteristic vibrations at $1601 \mathrm{~cm}^{-1}\left(\nu_{\mathrm{C}=\mathrm{C}}\right), 3323 \mathrm{~cm}^{-1}$ and $3208 \mathrm{~cm}^{-1}\left(\nu_{\mathrm{N}-\mathrm{H}}\right)$. The main characteristic peaks of GO are located at $3417 \mathrm{~cm}^{-1}$ $\left(\nu_{\mathrm{O}-\mathrm{H}}\right), 1736 \mathrm{~cm}^{-1}\left(\nu_{\mathrm{C}=\mathrm{O}}\right), 1435 \mathrm{~cm}^{-1}\left(\delta_{\mathrm{O}-\mathrm{H}}\right)$, and $1035 \mathrm{~cm}^{-1}$ $\left(\nu_{\mathrm{C}-\mathrm{O}}\right) \cdot{ }^{43}$ After aPcCu bonding to the surface of GO, the peak at $1736 \mathrm{~cm}^{-1}$ disappears, a new peak at $1651 \mathrm{~cm}^{-1}\left(\nu_{\mathrm{NH}-\mathrm{C}=\mathrm{O}}\right)$ appears. This result confirms the formation of an amido-bond between aPcCu and GO. Moreover, the characteristic fingerprint peaks of aPcCu also emerged at $800-1620 \mathrm{~cm}^{-1}$, providing a significant support for aPcCu covalently bonded onto GO. ${ }^{44}$ Fig. 1B shows the UV-vis spectra of GO, aPcCu and aPcCu-GO hybrid in DMF, respectively. A characteristic Q-band of aPcCu (719 $\mathrm{nm}$ ) observed in the UV-vis spectrum of the aPcCu-GO hybrid is broadened and blue shifts about $24 \mathrm{~nm}$, which is not only indicative of a covalently bonding between aPcCu and GO, but suggestive of ground-state electronic interactions between aPcCu and GO in hybrids. ${ }^{44,49}$ The covalent bonding of aPcCu-GO was also evidenced by Raman spectra. As shown in Fig. 1C, the Dand G-bands of the GO appear at 1361 and $1579 \mathrm{~cm}^{-1}$, respectively, which can be attributed to the defects introduced by the oxidation and the vibrations of the $\mathrm{sp}^{2}$ hybridized carbons in the 2-D lattice of graphite..$^{50}$ In contrast to the case of GO, the D- and G-bands of the aPcCu-GO hybrid, which appear at 1356 and 1570 $\mathrm{cm}^{-1}$, are found to be slightly shifted to higher energy region. ${ }^{43}$ The D- to G-band intensity ratios $\left(I_{\mathrm{D}} / I_{\mathrm{G}}\right)$ increased from 0.85 for GO to 0.98 for aPcCu-GO due to the formation of $\mathrm{sp}^{3}$ carbons by covalent attachment of aPcCu. ${ }^{44}$ The results indicate that $\mathrm{aPcCu}$ has succeeded in bonding onto the surface of GO.

As a further proof, XPS spectra provide an important and effective information for the covalent attachment of the aPcCu onto the GO. As shown in Fig. 2, in the survey spectrum of aPcCu-GO (Fig. 2A), $\mathrm{N}$ and $\mathrm{Cu}$ elements could be found. It further confirms successful adsorption of aPcCu derivatives onto the surface of GO. The high-resolution N 1s XPS spectrum of aPcCu-GO splits into three peaks located at $398.8 \mathrm{eV}$ (the $\mathrm{N}$ in $\mathrm{C}-\mathrm{N}$ bonds), $400.2 \mathrm{eV}$ (the $\mathrm{N}$ in $-\mathrm{NH}-\mathrm{CO}-$ bonds) and $401.2 \mathrm{eV}$ (the $\mathrm{N}$ in $\mathrm{C}=\mathrm{N}$ bonds) (Fig. 2B). The high-resolution $\mathrm{C} 1 \mathrm{~s}$ XPS spectrum of aPcCu shows that the peaks of carbon appear at $284.6 \mathrm{eV}$ (the $\mathrm{C}$ in $\mathrm{C}-\mathrm{C}$ bonds), $285.7 \mathrm{eV}$ (the $\mathrm{C}$ in $\mathrm{C}-\mathrm{O}$ bonds) and $287.7 \mathrm{eV}$ (the $\mathrm{C}$ in -NH-CO- bonds) (Fig. 2D). In contrast to aPcCu, the $\mathrm{N}$ peak in $\mathrm{NH}_{2}$ bonds blue shifts about $0.8 \mathrm{eV}$ and intensity of the peak increases significantly due to the electron withdrawing effect of GO. ${ }^{43}$ The binding energy of $\mathrm{Cu} 2 \mathrm{p}$ peak increases in the hybrid spectrum by $1.7 \mathrm{eV}$ compared to that in aPcCu (Fig. 2B). The binding energy in XPS spectrum is related to the electron density around the nucleus. The lower the electronic density is, the higher the binding energy will be, ${ }^{51}$ and therefore such higher binding energy shift is mainly attributed to the charge transfer of the aPcCu ring to the GO. ${ }^{52}$

The microstructures of the aPcM-GO hybrids were also characterized by SEM and TEM. The TEM images of GO were shown in Fig. $2 S . \dagger$ Fig. $2 \mathrm{~S} \dagger$ shows that the surface of GO is a few layers wrinkled paper-like structure. While aPcM-GO hybrid exhibits layered structure after covalent attachment of aPcCu onto the surface of GO, aPcCu islands are distributed on the GO surface without obvious large-scale aggregation of aPcCu (Fig. 3A). The typical SEM images of aPcM-GO sensors using a drop casting technique on interdigitated electrode surface are shown in Fig. 3B and C. As shown in Fig. 3B, aPcCu-GO hybrid displays few layered flakelike shape with a rounded morphology. Fig. 3C shows that aPcCu-GO sheets are evenly, loose distributed between the two-finger of interdigitated electrode, which provides continuous conducting pathways for transportation of electrons, the permeable channels for diffusion of gas molecules, and more advantages on the active sites, giving rise to the high sensing performance. ${ }^{53-55}$ The influence of aPcM molecules on the dispersion of the aPcM-GO hybrids in DMF was also studied. As shown in Fig. 3D-F, aPcM-GO hybrids can disperse in DMF well after sonicating at room temperature for 20 minutes, which is very beneficial to fabricating uniform films for using as gas sensors. The aPcM-GO hybrids remained as a good dispersion in addition to the aPcFe-GO hybrid settled down a little after standing for 15 days. More importantly, the aPcM-GO hybrids remained as a good dispersion even after
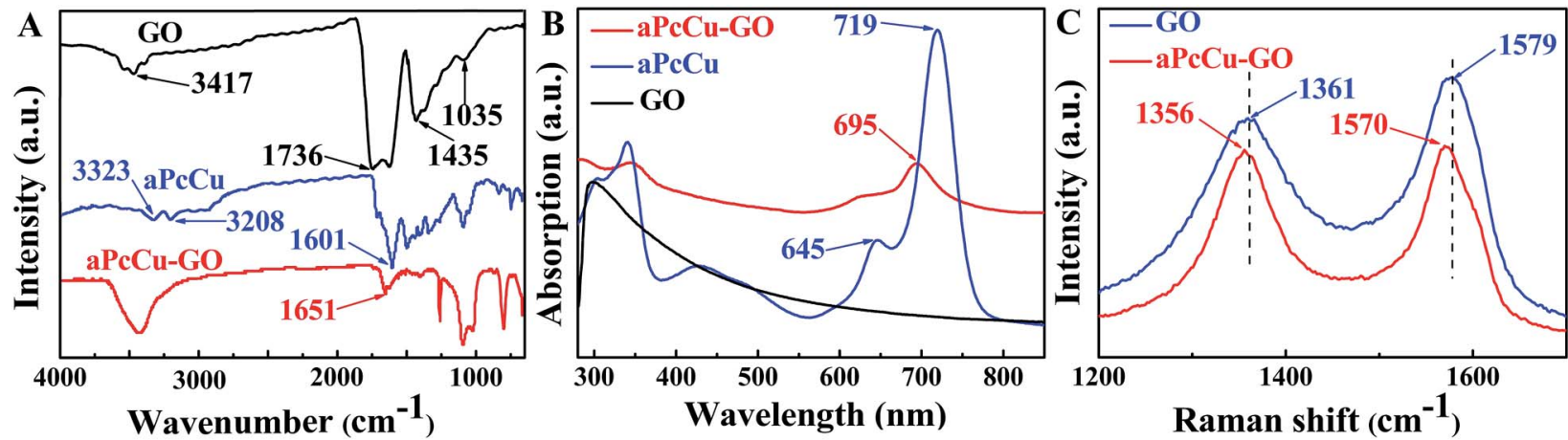

Fig. 1 (A) FT-IR spectra, (B) UV-vis spectra and (C) Raman spectra of GO and aPcCu-GO hybrid obtained at $\lambda_{\text {exc }}=457.9 \mathrm{~nm}$. 

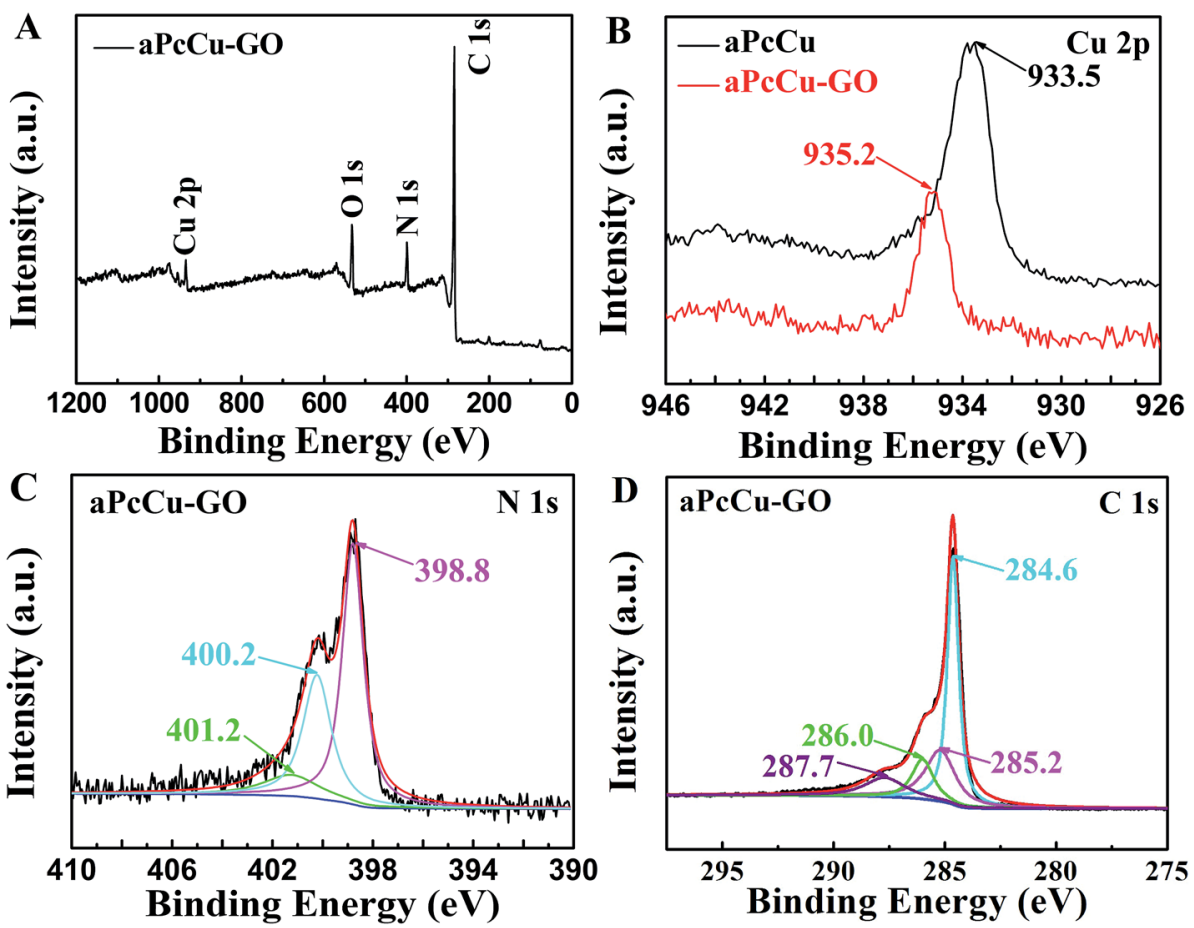

Fig. 2 XPS analysis of aPcCu-GO hybrids: (A) survey spectra; (B) N 1s regions; (C) Cu 2p region; (D) C 1s region.

standing for 30 days. Evidently, the long-term dispersion and stability of aPcM-GO can be attributed to the covalent bond of aPcM onto the surface of GO.

\section{2 $\mathrm{NH}_{3}$ sensing properties of aPcCu-GO hybrids}

To evaluate the $\mathrm{NH}_{3}$-sensing potential applicability in gas sensor, the $\mathrm{NH}_{3}$-sensing properties of aPcM-GO hybrids were studied. The resistance value of aPcM-GO hybrids sensors (about 0.1-0.2 M $\Omega$ ) also suggested that a perfect circuit of the sensing device had been achieved, which was about 2 times higher than pure GO sensor (about $6 \mathrm{k} \Omega$ ). For cPcCu-GO hybrids, the increase in sensor resistance is possibly caused by the intrinsically high resistance of aPcCu (about 30 000-40 000 $\mathrm{M} \Omega$ ), and the donation of electrons from the p-type aPcCu to the p-type GO, compared with aPcCu, the resistance of hybrid was

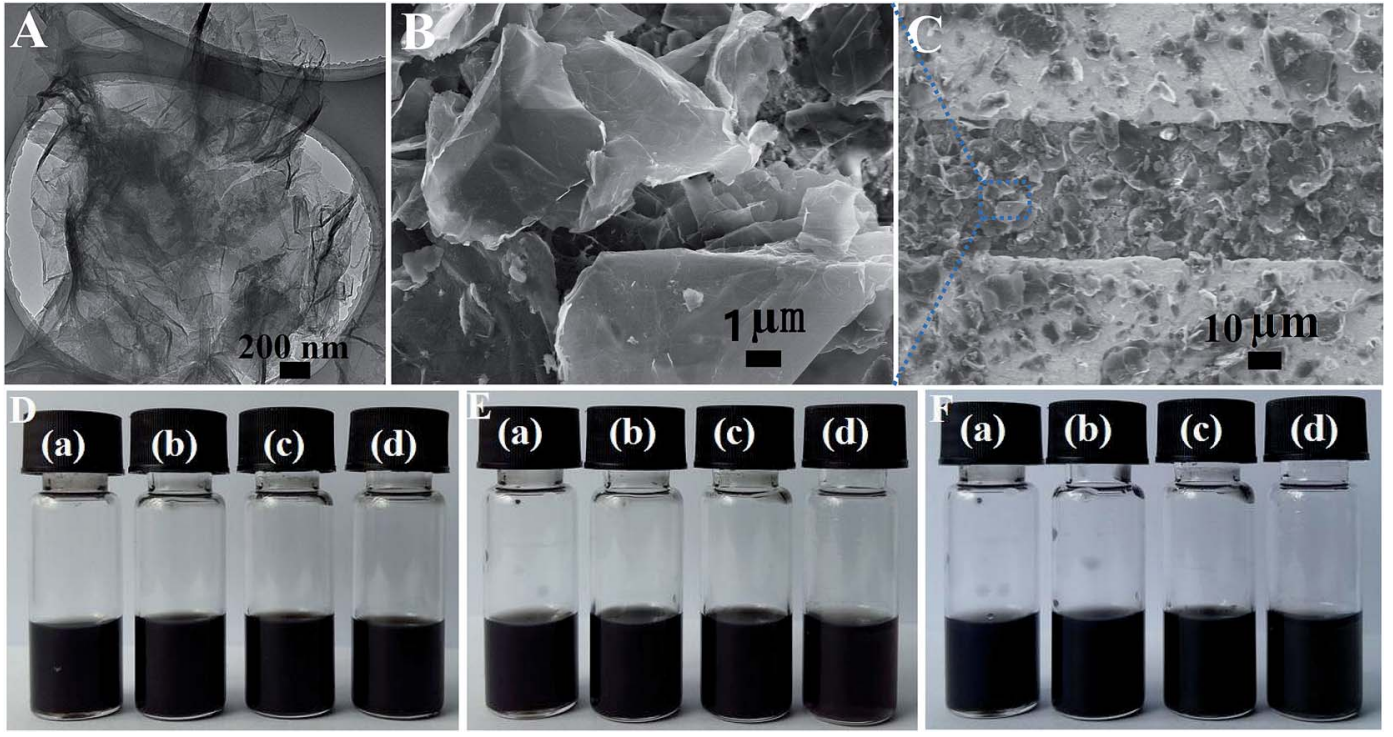

Fig. 3 (A) TEM images and (B and C) SEM images of aPcCU-GO; (D-F) optical photographs of the equivalent aPcM-GO hybrids dispersed in DMF after sonicating at room temperature for 20 minutes (D), and after standing at room temperature for 10 days (E) and 30 days (F). ((a-d): aPcCu$\mathrm{GO}, \mathrm{aPcCo}-\mathrm{GO}, \mathrm{aPcNi}-\mathrm{GO}, \mathrm{aPcFe}-\mathrm{GO}$, respectively). 
remarkably decreased, indicating the electrical conductivity of aPcM-GO hybrids was improved dramatically. It can be ascribed to the GO possess unique electrical properties, such as high electron mobility and low electrical noise. The improved conductivity and the specific compositions of semiconductor materials might be advantageous for gas-sensing applications.

To verify the potential application of the aPcCu-GO sensor, the selectivity of aPcCu-GO hybrid was studied. The representative gases in air and volatile organic compounds (VOCs) were separately injected into the test chamber in turns at room temperature (as shown in Fig. 4). Compared with GO sensor, aPcCu-GO sensor had excellent selectivity for $\mathrm{NH}_{3}$. This means that the covalent bonding of aPcCu on $\mathrm{GO}$ enhanced the selectivity of aPcCu-GO hybrid to $\mathrm{NH}_{3}$. Contrary to $\mathrm{NH}_{3}$, the resistance reduces sharply when aPcCu-GO sensor is exposed to the strong oxidants, e.g. $\mathrm{NO}_{x}$. It is because both aPcCu and $\mathrm{GO}$ are p-type semiconductors. $\mathrm{NO}_{x}$ can trap the electrons from aPcCu-GO hybrids and obtain more holes in hybrids, leading to the reduced resistance, which is consistent with the chargetransfer mechanism. Except of $\mathrm{NH}_{3}$ and $\mathrm{NO}_{x}$, aPcCu-GO sensor exhibiting negligible responses towards all these gases and VOCs even at very high concentrations of $3000 \mathrm{ppm}$, sensors also show very low response to different relative humidity. Therefore, it is a promising sensor to selectively detecting $\mathrm{NH}_{3}$ in an indoor atmosphere, industrial emission gas, agricultural practices gas, and so on.

The detailed dynamic resistance cure of the aPcCu-GO sensor upon repeated $\mathrm{NH}_{3}$ exposure/removal cycles are shown in Fig. 5A. Nine cycles were successively recorded at room temperature, corresponding to $\mathrm{NH}_{3}$ concentrations from $12.5 \mathrm{ppm}$ to $3200 \mathrm{ppm}$. The resistance rapidly increases upon $\mathrm{NH}_{3}$ gas, and the resistance decreases when aPcCu-GO sensor exposed to air again. Since both aPcCu and GO are p-type semiconductors, according to the charge transfer mechanism, $\mathrm{NH}_{3}$ can donate the electrons to aPcCu-GO hybrids and cause the depletion of holes in hybrids, leading to the increased resistance. The response intensities increase with the increasing concentration of $\mathrm{NH}_{3}$ gas. As a control, the sensing responses of $\mathrm{GO}$ and aPcM towards $\mathrm{NH}_{3}$ were also studied. Both the response intensity and the recovery properties are poor for the GO and aPcM sensors, (Fig. S3 $\dagger$ ). By contrast, the aPcCu-GO sensor exhibits remarkable response and recovery performance towards $\mathrm{NH}_{3}$. For example, the response of aPcCu-GO to $50 \mathrm{ppm} \mathrm{NH}_{3}$ is about $8.97 \%$ (Fig. $5 \mathrm{~B}$ ), which is over 30 times and 12 times higher than that of $\mathrm{GO}$ and aPcCu sensors, respectively; whereas, the recovery time of GO extends by $3450 \mathrm{~s}$ compared to that of aPcCu-GO (235 s). The aPcCu-GO sensor exhibits two good linear responses, $0.16 \%$ per ppm $\mathrm{NH}_{3}$ for concentrations ranging from $800 \mathrm{ppb}$ to $50 \mathrm{ppm}$, and $0.03 \%$ per ppm $\mathrm{NH}_{3}$ for concentrations ranging from 100 to $800 \mathrm{ppm}$ (Fig. 5B). The linear regression equation of $S=0.1600 \times C$ $(\mathrm{ppm})+1.3214\left(R^{2}=0.981\right)$ is thus derived from the calibration curve, revealing that the aPcCu-GO sensor has a limit of detection (LOD) as low as $800 \mathrm{ppb}(\mathrm{S} / \mathrm{N}=3)$.

To further verify the repeatability, reliability and stability, ten response cycles of the aPcCu-GO sensor to $50 \mathrm{ppm} \mathrm{NH}_{3}$ at room temperature were investigated (Fig. 5C). The resistance curve shows the similar continuous recycles, and there is no obvious change (less $3 \%$ ) in the ten continuous cycles. The sensor shows a fast recovery time (less than $250 \mathrm{~s}$ ). The long-term stability of the aPcCu-GO sensor was also evaluated over 30 days by determining the response to $50 \mathrm{ppm} \mathrm{NH}_{3}$ and storing in air at room temperature when not in use, and the results are shown in Fig. 5D. A slight response drop (only about 3\%) could be observed after storing for 30 days. Clearly, the aPcCu-GO sensor has good stability and repeatability, which is crucial for further applications. The high sensitivity, low detection limit and fast response and recovery time of our $\mathrm{NH}_{3}$ sensor are also significantly superior to the previously reported sensors (as shown in Table S1 $\dagger$ ).

Humidity is an important factor that might affect the $\mathrm{NH}_{3}$ sensing properties of aPcCu-GO sensor. Fig. 6 shows the response of aPcCu-GO hybrid sensor upon exposure to $50 \mathrm{ppm}$ $\mathrm{NH}_{3}$ in different relative humidity. As can be seen, the response of aPcCu-GO sensor did not increase obviously below $40 \% \mathrm{RH}$. In contrast, with further increasing $60 \% \mathrm{RH}$, the response increases significantly. However, the response decreases under the high $80 \% \mathrm{RH}$, which might be ascribed to the competitive adsorption between $\mathrm{NH}_{3}$ and $\mathrm{H}_{2} \mathrm{O}$ molecules. ${ }^{\mathbf{1 , 1 9}}$
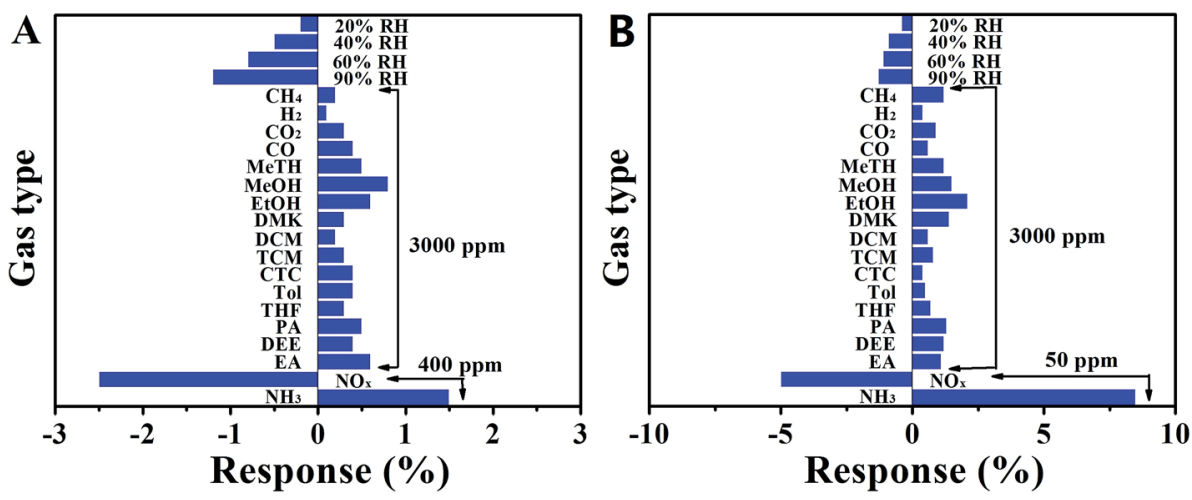

Fig. 4 Cross-sensitivities to various gases for the (A) $\mathrm{GO}$ and (B) aPcCu-GO sensor, $\mathrm{MeTH}=$ methanal, $\mathrm{MeOH}=\mathrm{methanol}$, EtOH $=$ ethanol, $\mathrm{DMK}=$ acetone, $\mathrm{DCM}=$ dichloromethane, $\mathrm{TCM}=$ trichloromethane, $\mathrm{CTC}=$ carbon tetrachloride, $\mathrm{Tol}=$ toluene, $\mathrm{THF}=$ tetrahydrofuran, $\mathrm{PA}=$ propionic acid, $\mathrm{DEE}=$ diethyl ether, $\mathrm{EA}=$ ethyl acetate. 

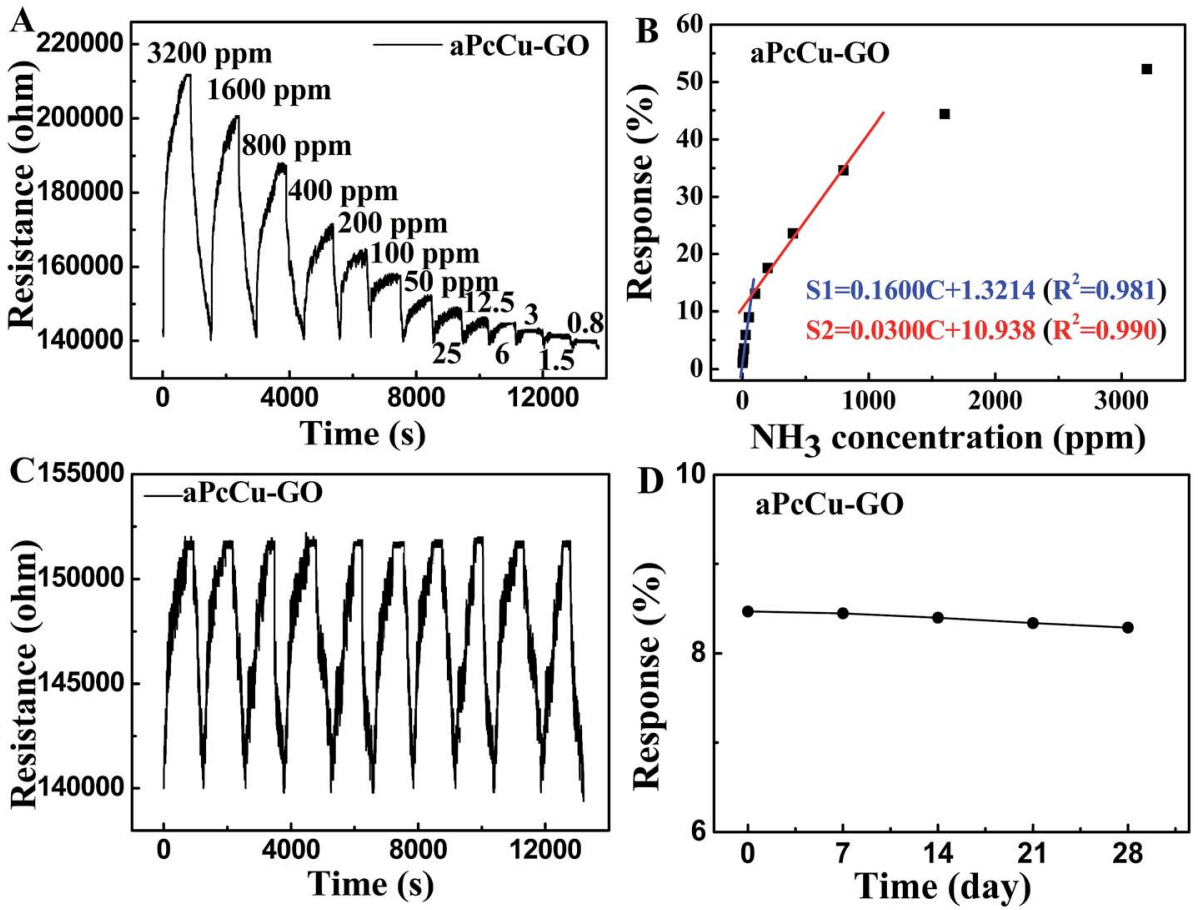

Fig. 5 (A) Resistance of aPcCu-GO hybrid sensor upon exposure to varying concentrations of $\mathrm{NH}_{3}$; (B) relationship of the response of aPcCu$\mathrm{GO}$ sensor to the concentration $\mathrm{NH}_{3}$; (C) ten sensing cycles of aPcCu-GO hybrid sensor to $50 \mathrm{ppm} \mathrm{NH}_{3}$; (D) response of aPcCu-GO hybrid sensors to $50 \mathrm{ppm} \mathrm{NH}$ over long time storage at $28^{\circ} \mathrm{C}$.

\subsection{The $\mathrm{NH}_{3}$-sensing mechanism of aPcM-GO hybrids}

Charge transfer can be responsible for the sensing mechanism of a PcCu-GO detecting $\mathrm{NH}_{3} \cdot{ }^{26,56,57}$ As shown in Scheme 2, GO and $\mathrm{aPcCu}$ show classic p-type behavior under ambient conditions, due to the electron withdrawing character of absorbed oxygen containing moieties which induce a hole-like carrier concentration. ${ }^{57} \mathrm{NH}_{3}$ is an electron donor with a lone electron pair. The exposure of hybrids to $\mathrm{NH}_{3}$ can cause a decrease in the number of charge carriers, resulting in an increase in resistance at room temperature, (Fig. 5A). During the sensing process, the electrons will transfer between the conjugated aPcCu and GO, and consequently increase the sensibility of the hybrids. This hypothesis is also responsible for the rapid increase of the resistance observed in Fig. 5A and S7. $\dagger$ Therefore, with the increase in the concentration of $\mathrm{NH}_{3}$, the response of aPcCu-

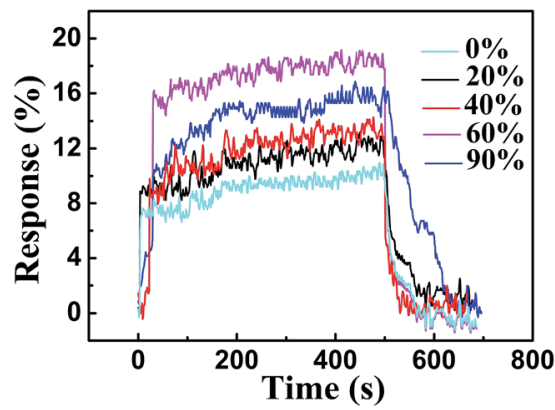

Fig. 6 Response of aPcCu-GO hybrid sensor upon exposure to $50 \mathrm{ppm} \mathrm{NH}$ in different relative humidity at $28^{\circ} \mathrm{C}$.
GO sensor increases. From all the above, the possible reasons for the excellent gas sensing property of aPcCu-GO hybrids can be ascribe to the following three factors. Firstly, the aPcCu covalently loaded on the surface of GO offers more sensing sites for $\mathrm{NH}_{3}$ reaction. Secondly, GO play a critical role in the charge transportation, and can accelerate the charge transfer as a conductive agent. Thirdly, the larger surface area, providing more active sites, is also a factor in the enhanced sensing property of aPcCu-GO to $\mathrm{NH}_{3}$. The enhanced $\mathrm{NH}_{3}$-sensing performance is mainly due to the synergistic effect between aPcCu and $\mathrm{GO}$, e.g. the stronger adsorption interaction of aPcCu with $\mathrm{NH}_{3}$, the higher electrical conductivity of GO, and a larger surface area of aPcCu-GO.

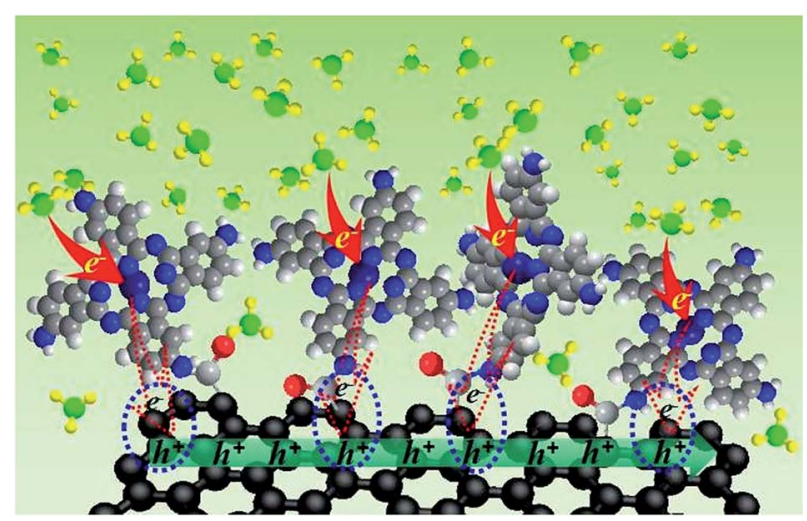

Scheme 2 A schematic illustration of the gas sensing mechanism of aPcM-GO sensors upon interaction with $\mathrm{NH}_{3}$. 
To validate the $\mathrm{NH}_{3}$ sensing mechanism, the $I-V$ characteristics of the aPcM-GO and aPcM-GO exposure to $\mathrm{NH}_{3}$ are investigated (Fig. S4†). The linearity and symmetry of $I-V$ curves suggest an ohmic contact between the samples and gold electrodes. Low current means the sensor has a relatively high resistance. For aPcM-GO hybrids, the decrease in device current is possibly caused by the intrinsically high resistance of aPcM, and the donation of electrons from the p-type aPcM to the p-type GO backbone. $\mathrm{NH}_{3}$ is an electron donor with a lone electron pair. The exposure of hybrids to $\mathrm{NH}_{3}$ can provide more electrons to combine with the holes in p-type semiconductors of aPcM and GO. The combination of electrons and holes can reduce the carrier concentration of the system, leading to further increase in the resistances of aPcM-GO hybrids exposure to $\mathrm{NH}_{3}$, which agrees well with the charge transfer mechanism. In addition, the excellent selectivity of aPcM-GO hybrids for $\mathrm{NH}_{3}$ can be mainly ascribed to the strong charge transfer interaction between $\mathrm{NH}_{3}$ molecules and the aPcM-GO hybrids. ${ }^{38}$

In general, the sensing effect mainly occur at aPcM in hybrids, which covalently loaded on the surface of GO. Most importantly, both theoretical and experimental evidence demonstrate that the central metals of PcMs are of primary importance to determine the selectivity and sensitivity, because of the preferential coordination with $\mathrm{NH}_{3},{ }^{58}$ whereas, GO play a critical role in the charge transportation..$^{23,42}$ To obtain further insight into it, various aPcM-GO hybrids ( $\mathrm{M}=\mathrm{Co}, \mathrm{Ni}$ and $\mathrm{Fe})$ were synthesized via covalent interaction. UV-vis, Raman, and XPS spectra further confirmed aPcM-GO hybrids have been successfully prepared (Fig. S5-S12†).
The resistance cure, reproducibility, stability, relationship of the response of aPcM-GO hybrids ( $\mathrm{M}=\mathrm{Co}, \mathrm{Ni}$ and $\mathrm{Fe}$ ) sensors to the concentration $\mathrm{NH}_{3}$ were also investigated (as shown in Fig. 7 and S13 and $14^{\dagger}$ ), and all aPcM-GO sensors exhibit more better response and recovery performance than GO, especially aPcCo-GO sensor, Fig. 7A and B shows that the response of aPcCo-GO to $50 \mathrm{ppm} \mathrm{NH}_{3}$ is about $11.6 \%$, which is over 40 times higher than that of GO sensor; whereas, the recovery time of GO extends by $3450 \mathrm{~s}$ compared to that of aPcCo-GO (350 s). The aPcCo-GO sensor exhibits also two good linear responses, $0.12 \%$ per $\mathrm{ppm} \mathrm{NH}_{3}$ for concentrations ranging from $800 \mathrm{ppb}$ to $50 \mathrm{ppm}$, and $0.03 \%$ per ppm $\mathrm{NH}_{3}$ for concentrations ranging from 100 to $400 \mathrm{ppm}$ (Fig. 7B). The linear regression equation of $S=0.1231 \times C(\mathrm{ppm})+4.7736\left(R^{2}=0.990\right)$ is thus derived from the calibration curve, revealing that the aPcCo-GO sensor has a limit of detection (LOD) as low as $800 \mathrm{ppb}(\mathrm{S} / \mathrm{N}=3)$. It's clear that these aPcM-GO sensors exhibit the distinct responses towards $\mathrm{NH}_{3}$ and good reproducibility, reliability and stability. The selectivity of aPcM-GO sensors to $\mathrm{NH}_{3}$ was also investigated (as shown in Fig. S15 $\dagger$ ). Similarly, to aPcCu-GO, other sensors also show good selectivity toward $\mathrm{NH}_{3}$ comparing to GO hybrids (Fig. 3S†), indicating that aPcM could enhance the $\mathrm{NH}_{3}$-sensing property due to the stronger adsorbing to $\mathrm{NH}_{3}$. More important, when the central metal of phthalocyanine is changed, the enhancement degree of aPcM-GO to different types of gases is different. As shown in Fig. 8, the response of various sensors decreases in the order of $\mathrm{Co}>\mathrm{Cu}>\mathrm{Ni}>\mathrm{Fe} \gg$ GO. The difference in responses are mainly due to the difference aPcM; or to be more specific, central metals, for example, the atomic radius,
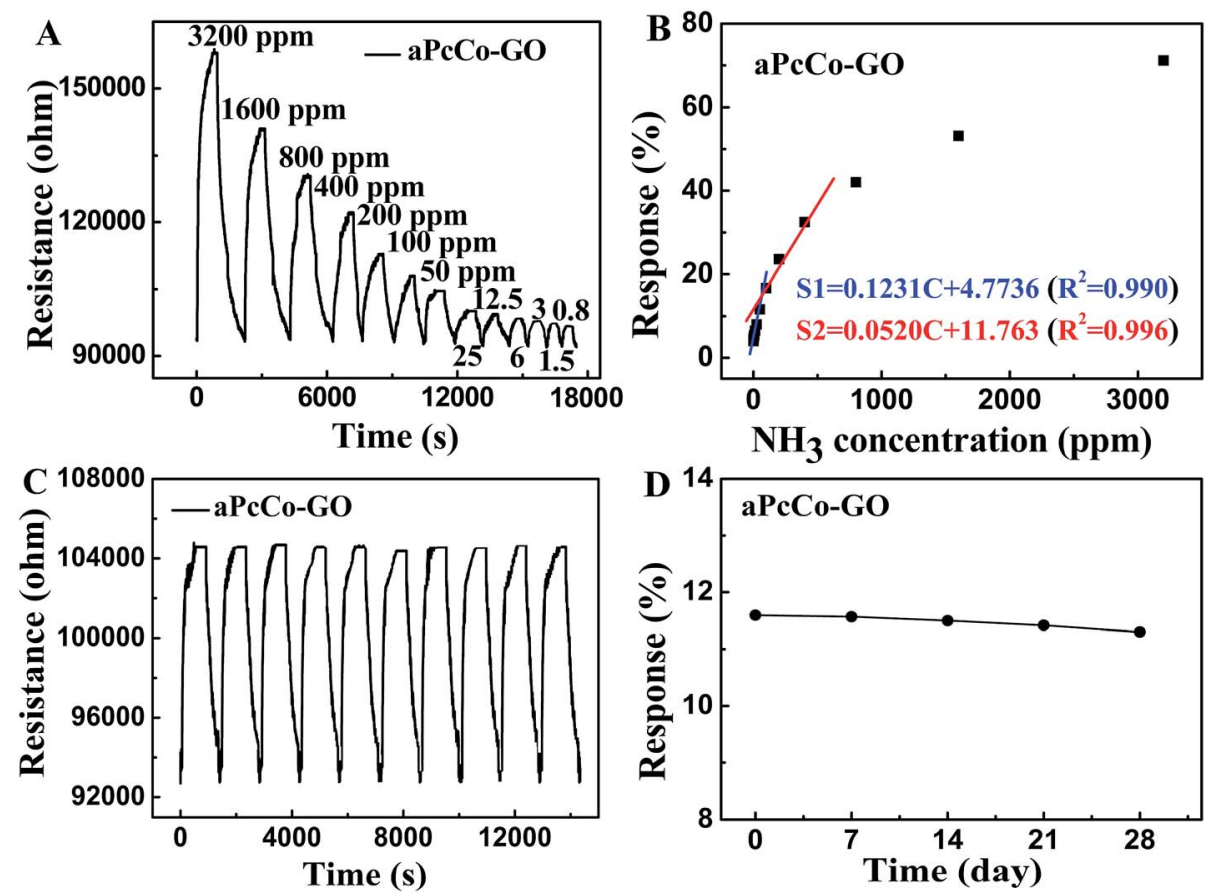

Fig. 7 (A) Resistance of aPcCo-GO hybrid sensor upon exposure to varying concentrations of $\mathrm{NH}_{3}$; (B) relationship of the response of aPcCo$\mathrm{GO}$ sensor to the concentration $\mathrm{NH}_{3}$; (C) ten sensing cycles of aPcCo-GO hybrid sensor to 50 ppm $\mathrm{NH}_{3}$; (D) response of aPcCo-GO hybrid sensors to $50 \mathrm{ppm} \mathrm{NH}$ over long time storage at $28^{\circ} \mathrm{C}$. 


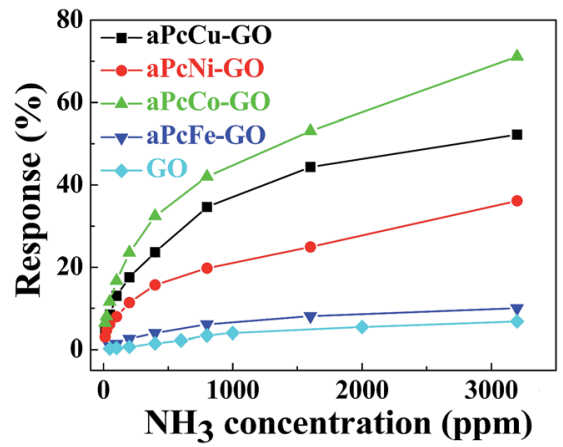

Fig. 8 Response of $\mathrm{GO}$ and $\mathrm{aPcM}-\mathrm{GO}$ hybrid sensors to different concentration of $\mathrm{NH}_{3}$ gas at $28^{\circ} \mathrm{C}$.

the valence state, the coordination number, especially the interaction effects between $\mathrm{NH}_{3}$ and different central ions.

Considering the structural properties of aPcM-GO hybrids, the same substitutes of aPcM, and less interaction of $\mathrm{NH}_{3}$ and GO, only the interactions between $\mathrm{NH}_{3}$ with the central metals of unsubstituted PcMs were investigated. To better understand the effects of center metals on $\mathrm{NH}_{3}$-sensing property of aPcMGO hybrids, detailed DFT calculations for $\mathrm{NH}_{3}-\mathrm{aPcM}$ were performed. Adsorption energies of $\mathrm{NH}_{3}$ on aPcMs, net charge on $\mathrm{NH}_{3}$ moiety, and distance between central metals and $\mathrm{NH}_{3}$ were listed in Table 1. Fig. S16, $\uparrow$ shows the structures of adsorbed $\mathrm{NH}_{3}-\mathrm{aPcM}$ with top and side views. As illustrated in Fig. S16, $\uparrow$ the $\mathrm{NH}_{3}$ is almost vertically adsorbed on the top of aPcMs with $\mathrm{N}$ atom bonding to the metals. The distance of nitrogen and metal atoms was shown in Table 1 ranges from 2.240 to $2.716 \AA$. The distance of nitrogen and metal atoms ranges from 2.240 to $2.716 \AA$ (Table 1 ) and is a simple index of the interaction strength. The longer distance is, the weaker of the interaction is, and vice versa. For example, the distance of $\mathrm{NH}_{3}-\mathrm{aPcNi}$ is longest and its adsorption energy of $\mathrm{NH}_{3}$ on aPcNi is smallest $\left(-3.6 \mathrm{kcal} \mathrm{mol}^{-1}\right)$. As can be seen, the adsorption energy of aPcM- $\mathrm{NH}_{3}$ are negative value, which illustrate the adsorption of $\mathrm{NH}_{3}$ on center metals are exothermic process, which will benefit the formation of stable structure. ${ }^{59}$ The adsorption energy of aPcCo- $\mathrm{NH}_{3}\left(-21.7 \mathrm{kcal} \mathrm{mol}^{-1}\right)$ are more negative than that of aPcCu, $\mathrm{Ni}, \mathrm{Fe}-\mathrm{NH}_{3}$, hence, $\mathrm{NH}_{3}$ shows affinity to interact on aPcCo-Go. To elucidate the charge transfer between the $\mathrm{NH}_{3}$ and aPcMs, NBO calculations were carried out. In general, the net charges on $\mathrm{NH}_{3}$ are positive

Table 1 Calculated adsorption energies, major bond distance, and net charges in the adsorption of $\mathrm{NH}_{3}$ on the $\operatorname{aPcMs}(\mathrm{M}=\mathrm{Co}, \mathrm{Cu}, \mathrm{Ni}, \mathrm{Fe})$

\begin{tabular}{llll}
\hline Sub. $^{a}$ & Net charge $^{b}$ & $d(\mathrm{M}-\mathrm{R})^{c}(\AA)$ & $\begin{array}{l}\text { Adsorption energy } \\
\left(\mathrm{kcal} \mathrm{mol}^{-1}\right)\end{array}$ \\
\hline CoPc & 0.162 & 2.240 & -21.7 \\
CuPc & 0.0986 & 2.356 & -12.4 \\
NiPc & 0.073 & 2.716 & -3.6 \\
FePc & 0.143 & 2.323 & -6.2
\end{tabular}

${ }^{a}$ The substrate. ${ }^{b}$ The net charge on $\mathrm{NH}_{3} \cdot{ }^{c}$ Distance between metal and $\mathrm{NH}_{3}$.
(Table 1), implying that there exists electron transfer from $\mathrm{NH}_{3}$ to aPcMs. Charge transfer indicates a charge transfer sensing mechanism for the aPcMs sensors, which agrees well with the experimental observation. It is noted that there is much charge transfer in the adsorption of $\mathrm{NH}_{3}$ on aPcCo $(0.162 e)$ than that of other systems, moreover, although there is no much charge transfer in the adsorption of $\mathrm{NH}_{3}$ on aPcNi $(0.073 e)$ and $\mathrm{aPcCu}$ (0.0986e), the electrons transfer from $\mathrm{NH}_{3}$ to $\mathrm{aPcM}(\mathrm{M}=\mathrm{Ni}$ and $\mathrm{Cu})$ will form a weak bound complex $\mathrm{M}^{+}\left(\mathrm{NH}_{3}\right)^{-}$due to electrostatic interaction and still indicates a charge transfer sensing mechanism. ${ }^{60}$ In short, the $\mathrm{NH}_{3}$ sensitivity of aPcCo-GO is the highest, further illustrating that central metals play a critical role in gas sensitivity.

\section{Conclusions}

In summary, we have demonstrated the fabrication and application of aPcM-GO hybrids for $\mathrm{NH}_{3}$ gas sensors at room temperature. aPcM-GO hybrids with good dispersibility were synthesized by covalent modification. Compared with many existing $\mathrm{NH}_{3}$ sensors, the obtained sensors, especially aPcCoGO sensor, show high sensitivity $\left(11.6 \%\right.$ to $50 \mathrm{ppm} \mathrm{NH}_{3}$ with a LOD of $800 \mathrm{ppb}$ ), excellent stability, reproducibility, fast response/recovery without any external stimulus, and pronounced selectivity towards $\mathrm{NH}_{3}$. In fact, the combination of GO and aPcM have greatly improved gas sensing performance for gas sensor application. In which GO provides high conductivity and the continuous pathways for charge transportation, aPcM possesses more active adsorption sites towards $\mathrm{NH}_{3}$ and plays a major role in gas sensing response. More importantly, the charge transfer interaction between aPcM and GO leads to very large and fast variation of the resistance. By comparison, the response of aPcM-GO hybrids to $\mathrm{NH}_{3}$ decreases gradually in the following order of $\mathrm{Co}>\mathrm{Cu}>$ $\mathrm{Ni}>\mathrm{Fe} \gg$ GO. DFT calculation reveals also that the central metal of aPcM plays a critical role in $\mathrm{NH}_{3}$ sensitivity, according to the bond length, binding energies and charge transfer of $\mathrm{MPc}-\mathrm{NH}_{3}$ system. The systematic study developed here provides a valid way to fabricate high-efficient $\mathrm{NH}_{3}$ sensors.

\section{Acknowledgements}

We gratefully acknowledgement financially supported from the National Natural Science Foundation of China (51202061, 51002046 and 21303030), the Natural Science Foundation for the Returned Overseas Scholars of Heilongjiang Province (LC2012C02), the Natural Science of Heilongjiang Province (B201308), the Research Fund of Heilongjiang Provincial Education Department (12511377 and 12521399), the Postdoctoral Science-Research Developmental Foundation of Heilongjiang Province (LBH-Q11015) and the Innovative Talents Program for the Returned Overseas Scholars of Harbin (2012RFLXG031), the Opening Project Foundation of Key Laboratory of Functional Inorganic Material Chemistry (Heilongjiang University), Ministry of Education. 


\section{References}

1 C. H. Liu, H. L. Tai, P. Zhang, Z. B. Ye, Y. J. Su and Y. D. Jiang, Sens. Actuators, B, 2017, 246, 85.

2 M. Eising, C. E. Cava, R. V. Salvatierra, A. J. G. Zarbinc and L. S. Roman, Sens. Actuators, B, 2017, 245, 25.

3 L. Tabrizi and H. Chiniforoshan, Sens. Actuators, B, 2017, 245, 815.

4 S. M. Cui, H. H. Pu, G. H. Lu, Z. H. Wen, E. C. Mattson, C. J. Hirschmugl, M. Gajdardziska-Josifovska, M. Weinert and J. H. Chen, ACS Appl. Mater. Interfaces, 2012, 4, 4898.

$5 \mathrm{NIOSH}$ pocket guide to chemical hazards, September 2007, NIOSH Publ. No 2005-149, http://www.cdc.gov/niosh/docs/ 2005-149.

6 F. Shao, F. Hernández-Ramíreza, J. D. Prades, C. Fàbrega, T. Andreu and J. R. Morante, Appl. Surf. Sci., 2014, 311, 177.

7 J. Wang, L. M. Wei, L. Y. Zhang, C. H. Jiang, E. S.-W. Kong and Y. F. Zhang, J. Mater. Chem., 2012, 22, 8327.

8 M. D'Arienzo, L. Armelao, C. M. Mari, S. Polizzi, R. Ruffo, R. Scotti and F. Morazzoni, J. Am. Chem. Soc., 2011, 133, 5296. 9 H. Malkeshi and H. M. Moghaddam, J. Polym. Res., 2016, 23, 1.

10 T. Mérian, N. Redon, Z. Zujovic, D. Stanisavljev, J. L. Wojkiewicz and M. Gizdavic-Nikolaidis, Sens. Actuators, B, 2014, 203, 626.

11 M. Matsuguchi and T. Asahi, Sens. Actuators, BSens. Actuators, B, 2011, 160, 999.

12 J. W. Han, B. Kim, J. Li and M. Meyyappan, RSC Adv., 2014, 4, 549.

13 R. Ghosh, A. Midya, S. Santra, S. K. Ray and P. K. Guha, ACS Appl. Mater. Interfaces, 2013, 5, 7599.

14 F. Yavari, E. Castillo, H. Gullapalli, P. M. Ajayan and N. Koratkar, Appl. Phys. Lett., 2012, 100, 203120.

15 K. A. Mirica, J. G. Weis, J. M. Schnorr, B. Esser and T. M. Swager, Angew. Chem., Int. Ed., 2012, 51, 10740.

16 Q. X. Feng, X. G. Lia and J. Wang, Sens. Actuators, B, 2017, 243, 1115.

17 Y. L. Tang, Z. J. Li, X. T. Zu, J. Y. Ma, L. Wang, J. Yang, B. Du and Q. K. Yu, J. Hazard. Mater., 2015, 298, 154.

18 L. L. Wang, H. Huang, S. H. Xiao, D. P. Cai, Y. Liu, B. Liu, D. D. Wang, C. X. Wang, H. Li, Y. R. Wang, Q. H. Li and T. H. Wang, ACS Appl. Mater. Interfaces, 2014, 6, 14131.

19 H. Song, X. Li, P. Cui, S. X. Guo, W. H. Liu and X. L. Wang, Sens. Actuators, B, 2017, 244, 124.

20 Z. P. Liu, G. M. Liao, S. Y. Li, Y. Y. Pan, X. Y. Wang, Y. Y. Weng, X. H. Zhang and Z. H. Yang, J. Mater. Chem. A, 2013, 1, 13321.

21 N. T. Hu, Y. Y. Wang, J. Chai, R. G. Gao, Z. Wang, E. S. W. Kong and Y. F. Zhang, Sens. Actuators, B, 2012, 163, 97.

22 N. T. Hu, Z. Yang, Y. Y. Wang, L. L. Zhang, Y. Wang, X. L. Huang, H. Wei, L. M. Wei and Y. F. Zhang, Nanotechnology, 2014, 25, 025502.

23 S. S. Varghese, Su. Lonkar, K. K. Singh, S. Swaminathan and A. Abdala, Sens. Actuators, B, 2015, 218, 160.
24 S. Mao, G. H. Lu and J. H. Chen, J. Mater. Chem. A, 2014, 2, 5573.

25 W. J. Yuan and G. Q. Shi, J. Mater. Chem. A, 2013, 1, 10078. 26 E. Llobet, Sens. Actuators, B, 2013, 179, 32.

27 Y. Peng and J. H. Li, Front. Environ. Sci. Eng., 2013, 7, 403.

28 Y. Kim, T. K. An, J. Kim, J. Hwang, S. Park, S. Nam, et al., J. Mater. Chem. C, 2014, 2, 4539.

29 G. Singh, A. Choudhary, D. Haranath, A. G. Joshi, N. Singh, S. Singh, et al., Carbon, 2012, 50, 385.

30 X. Y. Li, X. D. Chen, Y. Yao, N. Li, X. P. Chen and X. Bi, IEEE Sens. J., 2013, 13, 4749.

31 X. M. Ding, H. J. Xu, L. G. Zhang, D. P. Jiang and A. D. Lu, Mol. Cryst. Liq. Cryst., 1999, 337, 481.

32 D. P. Jiang, A. D. Lu, Y. J. Li, X. M. Pang and Y. L. Hua, Thin Solid Films, 1991, 199, 173.

33 F. I. Bohrer, C. N. Colesniuc, J. Park, M. E. Ruidiaz, I. K. Schuller, A. C. Kummel and W. C. Trogler, J. Am. Chem. Soc., 2009, 131, 478.

34 T. Sizun, M. Bouvetn and J. Suisse, Talanta, 2012, 97, 318. 35 X. J. Wang, S. L. Ji, H. B. Wang and D. H. Yan, Sens. Actuators, $B, 2011,160,115$.

36 A. Singh, S. Samanta, A. Kumar, A. K. Debnath, R. Prasad, P. Veerender, V. Balouria, D. K. Aswal and S. K. Gupta, Org. Electron., 2012, 13, 2600.

37 B. Wang, X. Q. Zhou, Y. Q. Wu, Z. M. Chen, C. Y. He and X. Zuo, Sens. Actuators, B, 2012, 161, 498.

38 X. H. Liang, Z. M. Chen, H. Wu, L. X. Guo, C. Y. He, B. Wang and Y. Q. Wu, Carbon, 2014, 80, 268.

39 H. Wu, Z. M. Chen, J. L. Zhang, F. Wu, C. Y. He, B. Wang, Y. Q. Wu and Z. Y. Ren, J. Mater. Chem. A, 2016, 4, 1096.

40 Y. Y. Wang, N. T. Hu, Z. H. Zhou, D. Xu, Z. Wang, Z. Yang, H. Wei, E. S. W. Kong and Y. F. Zhang, J. Mater. Chem., 2011, 21, 3779.

41 B. Wang, Y. Q. Wu, X. L. Wang, Z. M. Chen and C. Y. He, Sens. Actuators, B, 2014, 190, 157.

42 X. Q. Zhou, X. L. Wang, B. Wang, Z. M. Chen, C. Y. He and Y. Q. Wu, Sens. Actuators, B, 2014, 193, 340.

43 J. H. Zhu, Y. X. Li, Y. Chen, J. Wang, B. Zhang, J. J. Zhang and W. J. Blau, Carbon, 2011, 49, 1900.

44 N. Karousis, J. Ortiz, K. Ohkubo, T. Hasobe, S. Fukuzumi, Á. Sastre-Santos and N. Tagmatarchis, J. Phys. Chem. C, 2012, 116, 20564.

45 M. Ragoussi, J. Malig, G. Katsukis, B. Butz, E. Spiecker, G. Torre, T. Torres and D. M. Guldi, Angew. Chem., Int. Ed., 2012, 51, 6421.

46 T. Yanaia, D. P. Tew and N. C. Handyb, Chem. Phys. Lett., 2004, 393, 51.

47 E. D. Glendening, A. E. Carpenter and F. Weinhold, NBO, version 3.1, 1995.

48 M. J. Frisch, et al., Gaussian 09, Revision B01, Gaussian, Inc., Wallingford, CT, 2009.

49 W. Chidawanyika and T. Nyokong, Carbon, 2010, 48, 2831. 50 N. Karousis, A. S. D. Sandanayaka, T. Hasobe, S. P. Economopoulos, E. Sarantopoulou and N. Tagmatarchis, J. Mater. Chem., 2011, 21, 109.

51 L. Cao, H. Chen, M. Wang, J. Sun, X. Zhang and F. Kong, J. Phys. Chem. B, 2002, 106, 8971. 
52 A. Chunder, T. Pal, S. I. Khondaker and L. Zhai, J. Phys. Chem. C, 2010, 114, 15129.

53 F. D'Souza and O. Ito, Chem. Commun., 2009, 4913.

54 P. Li, Y. Ding, A. Wang, L. Zhou, S. H. Wei, Y. M. Zhou, Y. W. Tang, Y. Chen, C. X. Cai and T. H. Lu, ACS Appl. Mater. Interfaces, 2013, 5, 2255.

55 P. Li, H. L. Liu, J. Yang, D. M. Sun, Y. Chen, Y. M. Zhou, C. X. Cai and T. H. Lu, J. Mater. Chem. B, 2013, 179, 32.

56 J. Zhang, X. H. Liu, G. Neri and N. Pinna, Adv. Mater., 2016, 28, 795 .
57 X. L. Huang, N. T. Hu, R. G. Gao, Y. Yu, Y. Y. Wang, Z. Yang, et al., J. Mater. Chem., 2012, 22, 22488.

58 F. I. Bohrer, C. N. Colesniuc, J. Park, M. E. Ruidiaz, I. K. Schuller, A. C. Kummel and W. C. Trogler, J. Am. Chem. Soc., 2009, 131, 478.

59 Z. F. Cao, Q. B. Chen, Y. X. Lu, H. L. Liu and Y. Hu, Int. J. Quantum Chem., 2013, 113, 1137.

60 X. Zhou, W. Q. Tian and X. L. Wang, Sens. Actuators, B, 2010, 151, 56. 